\title{
BLOOD FLOW ANALYSIS OF POLYURETHANE HEART VALVES AT PEAK SYSTOLE
}

\author{
Cheung-Hwa Hsu \\ Department of Mechanical Engineering, \\ Southern Taiwan University of Technology, Tainan, Taiwan
}

\begin{abstract}
Polyurethane (PU) heart valves provide central flow at peak systole and the associated hemodynamic characteristics are superior to that of mechanical valves with almost no anticoagulation therapy for patients. Durability performances, on the other hand, are also superior to those of biological valves. This paper analyzes blood flow characteristics of the PU heart valves at fully open position with computational fluid dynamics. These data provide information for the improvement of leaflets and leaflet support geometry to minimize the scale of recirculation zone of the flow field. To simulate the hemodynamic characteristics of the blood flow, CFX-4.3 software with the finite volume method is utilized to analyze the three-dimensional Reynolds-averaged NavierStokes equations. By modifying the geometry of leaflets along with the supports, the scale of vortex flow and blood velocity are reduced obviously. Maximum flow velocity reduces 33\% compared to that of original model at peak systole.
\end{abstract}

Biomed Eng Appl Basis Comm, 2006(February); 18: 13-18.

Keywords: polyurethane heart valve, peak systole, computational fluid dynamics

\section{INTRODUCTION}

Functioning as a check valve, heart valves control blood's inflow or outflow of the heart at circulatory system. As the heart valve lacks proper care because of pathological change or congenital function, it is not able to completely close and cause regurgitation, or the valve is narrow and unable to totally open and causes blood flow obstruction to increase loads of the heart. The clinical surgery for these patients will often replace the heart valve by the operation of artificial heart valve replacement.

No kinds of artificial heart valve can act perfectly as human body's nature valve now, even if the widest

Received: Sept. 6, 2005; Accepted: Jan. 10, 2006

Correspondence: Cheung-Hwa Hsu, Professor

Department of Mechanical Engineering, Southern Taiwan University of Technology, 1 Nan-Tai Street, Yung-Kang City, Tainan, Taiwan

E-mail: chhsu@mail.stut.edu.tw used St. Jude Medical mechanical valve often criticized with the drawback of thrombus formation. The patient must still take the blood coagulation drug for a long time. On the other hand, the biological valve, although owns excellent hemodynamic characteristics while is often criticized with poor durability and often need second operation. The newly developed PU valve is therefore developed to keep both the advantages of mechanical ones and that of biological ones. Its leaflet is manufactured with medical polyurethane and therefore owns excellent hemodynamic characteristic as the valve leaflet is flexible while the durability, on the other hand, is much better than that of biological valve. This research concentrates on PU heart valve at peak systole and analyzes its hemodynamic performance by computational fluid dynamics. Utilizing a three dimensional numerical model, we analyze PU aortic valve's blood flow field when leaflet at fully opened 
position at peak systole. The purpose here is to highlight the correlation between the design valve geometry of both valve leaflet and support with that of blood flow performance. This would offer better valve designs and reference material for the clinical experiment in the future.

\section{METHOD}

\subsection{Geometric Modeling and Surface Generation of PU valve}

The geometry of the newly developed PU valve is generated by the partial differential equations (PDEs) method developed by Bloor and Wilson [1]. With the method by choosing appropriate boundary conditions, the solutions to suitably chosen elliptic partial differential equations can define exactly the desired shape and its surface properties. Thus, by defining the boundary curves of a patch of surface and by varying the derivative conditions imposed upon them, it is possible to create a wide variety of shapes. The behavior of PDEs surfaces is controlled by the boundary conditions that can readily be given a geometric interpretation, and parameterized in terms of a small number of variables. This makes the PDEs method distinctly superior from the conventional methods.

Consider the fourth-order elliptic partial differential equation,

$$
\begin{gathered}
\left(\frac{\partial^{2}}{\partial u^{2}}+a \frac{\partial^{2}}{\partial v^{2}}\right)^{2} \mathbf{X}(u, v)=0 \\
\mathbf{X}(u, v)=(x(u, v), y(u, v), z(u, v))
\end{gathered}
$$

A heart valve geometry design can be created by appropriately choosing the associated Dirichlet and Neumann boundary conditions.

\subsection{Visualization Environment}

Solutions from the PDEs method are viewed in the visualization tool, named Iris Explorer. The Iris Explorer provides a graphic framework, called Modular Visualization Environment (MVE), for investigating scientific data. In MVE, users create visualization maps composed of modules interlinked with a data-flow paradigm to process input data and user interaction into numerical or graphic output. Hsu [2] has utilized the PDEs method and MVE to develop an interactive and integrated design environment for mechanical heart valves. In this paper, applications have been extended for the design works of PU aortic valve.

\subsection{Numerical Flow Analysis}

We adopt the finite volume methods software CFX4.3 to investigate the corresponding flow phenomena. To simplify the analysis, we assume uniform velocity distribution at inlet plane. Only axial velocity is considered and its magnitude is determined from the design flow-rate. At the outlet plane, known pressure is specified. No slip and no-flux boundary conditions are imposed on all solid surfaces. Inlet conditions for turbulent kinetic energy $k$ and turbulent dissipation rate $\varepsilon$ are established using the empirical relations [3],

$$
\begin{aligned}
& k_{\text {in }}=0.05 \frac{|\vec{V}|^{2}}{2} \\
& \varepsilon_{i n}=\frac{c_{\mu} \cdot k_{i n}^{1.5}}{0.01 \cdot L}
\end{aligned}
$$

where $\vec{V}$ is the velocity at the inlet plane. $L$ is the characteristic. Solutions are also obtained by arbitrarily varying of $k$ and $\varepsilon$. The flow patterns within the channel are found to be independent of the assumed turbulent parameters at the inlet. The turbulence generated by overall flow field dominates the flow behavior within the channel rather than the turbulent parameters upstream to the inflow orifice.

\section{RESULT AND DISCUSSION}

\subsection{Physical Model}

This research utilizes three-dimensional geometry model to simulate blood flows from the left ventricle through the artificial heart valve into the aorta. Leaflet data refers to that of Leeds polyurethane tri-leaflet valve (LPTV) [4] to stick in three thin supports. The open angle of leaflet at fully open position is about 70 degree. Fig. 1 is a geometry appearance of this research simulation.

\subsection{Valve Support Geometry Design}

The support of the valve leaflets is integrated with sewing ring and is usually designed with very thin geometry. We have developed two PU aortic valve models with supports of different support heights, and named as model-1 of support height $4.25 \mathrm{~mm}$ and model-2 of support height $7.45 \mathrm{~mm}$.

\subsection{Model-1 Analysis Results}

To determine mesh density required for simulation with enough accuracy, we pursue a case of peak volume flow rate of $380 \mathrm{ml} / \mathrm{s}$ with different mesh densities and discuss the density required. We adopt 
mesh density with mesh seed of 4, 5 and 6 . It has been found that all the flow characteristics are very similar that the largest velocity of flow takes place near the sewing ring entrance. We then compare calculated axial velocity at the entrance A-A section as shown in Fig.2. The calculated axial velocity results form three density are rather consistent. We adopt mesh density with mesh seed 4 as an index in considering a balance between the simulation accuracy and required $\mathrm{CPU}$ time.

Model-1 valve leaflet support's height is designed

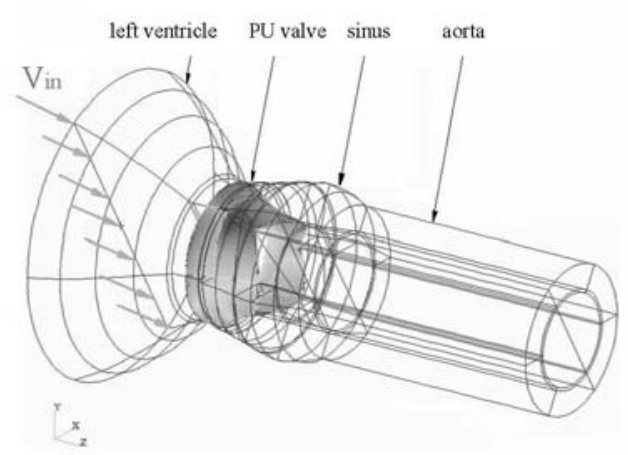

Fig 1. Geometry appearance of flow domain

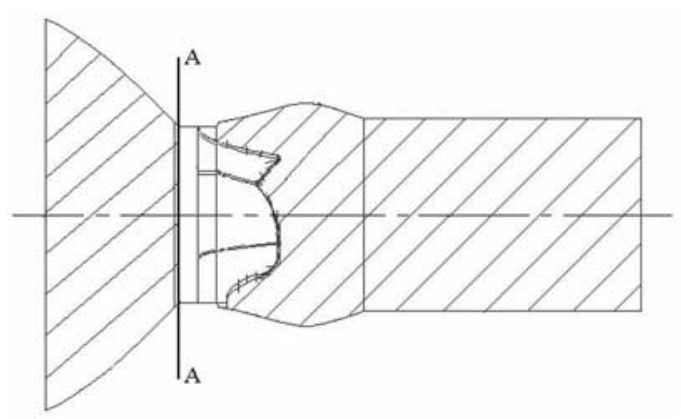

(a) A-A section

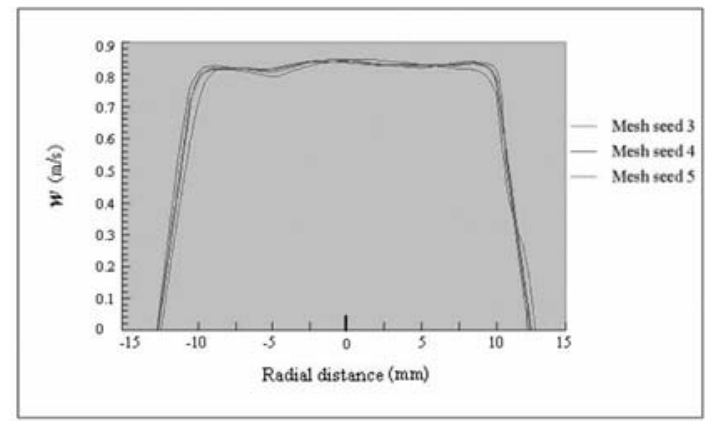

(b) Calculated axial velocity

Fig 2. Calculated axial velocity at sewing ring entrance A-A section of $4.25 \mathrm{~mm}$ and the inlet velocity of blood flow is $0.139 \mathrm{~m} / \mathrm{s}$ when volume flow rate at peak value of $380 \mathrm{ml} / \mathrm{s}$. From velocity distribution of model-1 (Fig.3), we can observe there is no violent changes of the velocity in left ventricle inlet and blood flow accelerates towards sewing ring inlet and the velocity of flow at that location lies between $0.1716 \sim 0.4005 \mathrm{~m} / \mathrm{s}$. Velocity at upstream of sewing ring reaches $0.5722 \sim 0.6294 \mathrm{~m} / \mathrm{s}$ and at downstream of sewing ring flow reaches the largest range of $1.087 \sim 1.144 \mathrm{~m} / \mathrm{s}$. Velocity in the aorta lies between 0.2861 and $0.5722 \mathrm{~m} / \mathrm{s}$. The stream-line distribution (Fig.4) shows that there are obvious vortex produced somewhere in the sinus especially in leaflet arc bottom and the region between the sinus wall and leaflet back.

In view of model-1 velocity field, there has already grown reversed flow between the walls in the support top and leaflet back. In order to imitate the greatest reversed velocity behaving in this phenomenon, we analyze the flow region of reversed speed and predict the strength of vortex evolved.

With table I and the corresponding Fig.5 of

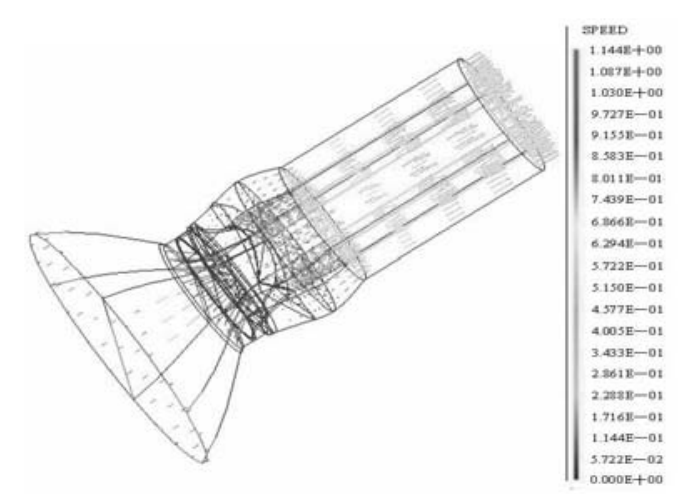

Fig 3. Velocity distribution of model-1 PU aortic valve

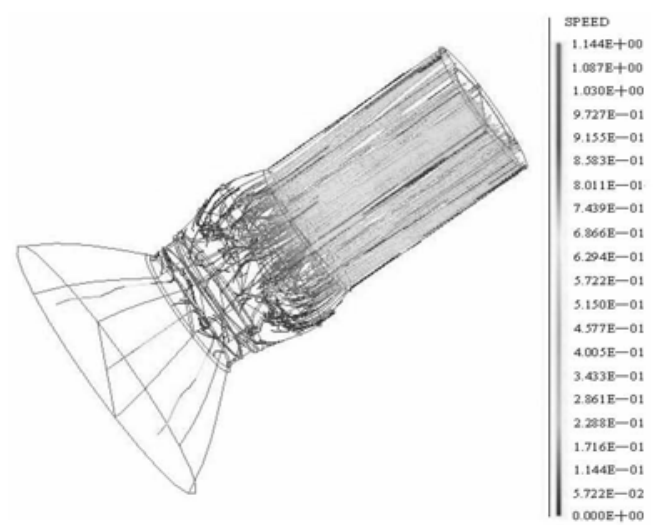

Fig 4. Streamline distribution of model-1 PU aortic valve 
Table I Range of axial velocities of model-1 PU aortic valve at different sections

\begin{tabular}{|c|c|c|}
\hline Distance from inlet $(\mathrm{mm})$ & Maximum $w$ value $(\mathrm{m} / \mathrm{s})$ & Minimum $w$ value $(\mathrm{m} / \mathrm{s})$ \\
\hline 27.0 & $0.0000000 \mathrm{E}+00$ & $-4.0570805 \mathrm{E}-03$ \\
\hline 31.6 & $3.6576804 \mathrm{E}-02$ & $-3.3742160 \mathrm{E}-02$ \\
\hline 32.0 & $6.4137511 \mathrm{E}-02$ & $-4.1255165 \mathrm{E}-02$ \\
\hline 33.0 & $6.9738768 \mathrm{E}-02$ & $-7.0037665 \mathrm{E}-02$ \\
\hline 35.0 & 0.1504384 & $-1.5822520 \mathrm{E}-02$ \\
\hline 39.0 & 0.1064373 & $-2.7588519 \mathrm{E}-06$ \\
\hline 40.0 & 0.1149636 & $1.1345307 \mathrm{E}-06$ \\
\hline 47.7 & 0.2287638 & $1.7904006 \mathrm{E}-07$ \\
\hline
\end{tabular}

schematic diagram of different locations, we find that the reversed vortex takes place somewhere $27 \sim 39 \mathrm{~mm}$ downstream the left ventricle entrance. The greatest reversed velocity lies in the section of $33 \mathrm{~mm}$ downstream of the left ventricle entrances and the velocity $w$ equals $-0.07 \mathrm{~m} / \mathrm{s}$.

Flow field analysis of model-1 PU aortic valve shows the trend that the flow field performs great change in sewing ring area and induces recirculation zone in the leaflet support top and leaflet back. In the aorta area, velocity field and the streamlines are comparatively steady and parallel to aortic wall. Centrifugal force produced of the vortex flow will not merely make the blood platelet keep in touch with endothelial cells, increase the blood coagulation factor but also cause tangible compositions and damage endothelial cell. All these could stimulate blood coagulation mechanism and cause the forming of thrombus. So this research attempts to improve the height of the leaflet support and leaflet geometry to reduce the possibility of vortex induced and blood coagulation.

\subsection{Model-2 Analysis Results}

According to the analysis result and in order to minimize the vortex induced, we attempt to do some changes of leaflet support geometry and design a new PU aortic valve, named model-2, with support height of $7.45 \mathrm{~mm}$. We also revise leaflet arc bottom and make leaflet open with a larger area at the same time.

The entry velocity for volume flow rate of $380 \mathrm{ml} / \mathrm{s}$ is $0.139 \mathrm{~m} / \mathrm{s}$ at peak systole. Pursued velocity field (Fig.6), we can observe that it is all steady and blood flows towards the sewing ring with no violent change of flow field. Speed nearly lies between $0.1630 \sim 0.4348 \mathrm{~m} / \mathrm{s}$ in the ventricle entry region. Blood flows toward the sewing ring with smooth-going stability and the speed drops to $0.3804 \sim 0.434 \mathrm{~m} / \mathrm{s}$
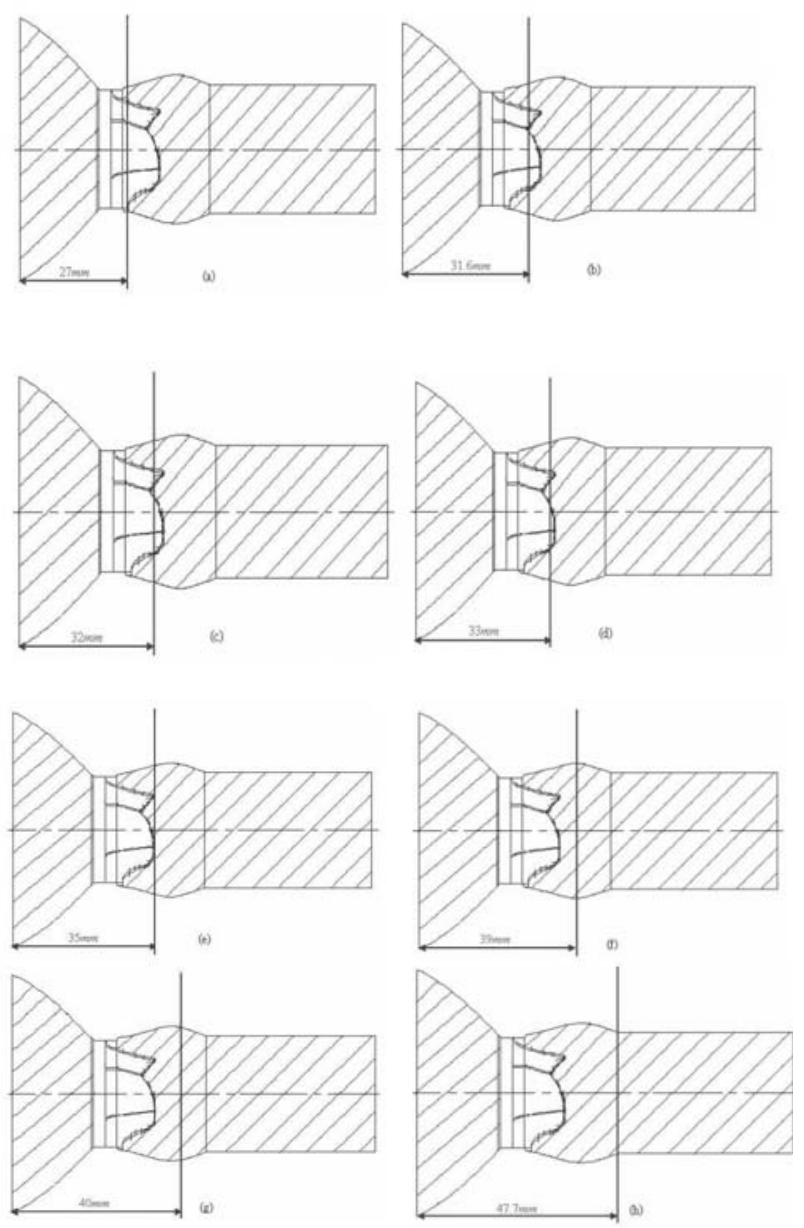

Fig 5. Schematic diagram of different sections corresponding to table I

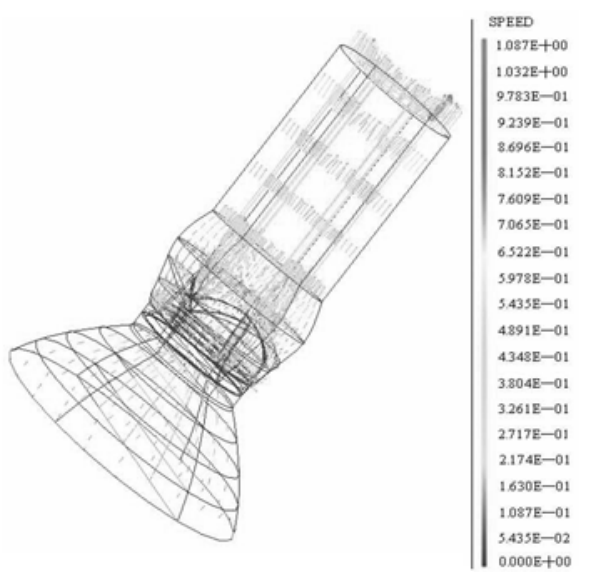

Fig 6. Velocity distribution of model-2 PU aortic valve 
roughly. Downstream the sewing ring, velocity lies in the range of $0.4891-0.5435 \mathrm{~m} / \mathrm{s}$. Streamline shows very steady too (Fig.7) and there is no vortex produced. Flow velocity lies between $0.6522 \sim 0.7609 \mathrm{~m} / \mathrm{s}$ steadily while flowing through the aorta.

In order to gain insight the flow region around the leaflet and the sinus, we probe into the calculated velocity at the same section of distance $27 \sim 47.7 \mathrm{~mm}$ downstream from the left ventricle entrance as the previous model. The calculated axial velocity is shown in Table II. It is found that there is no obvious vortex phenomenon in the whole flow field. So the improved model-2 geometry can effectively reduce the possibility of inducing vortex flow field.

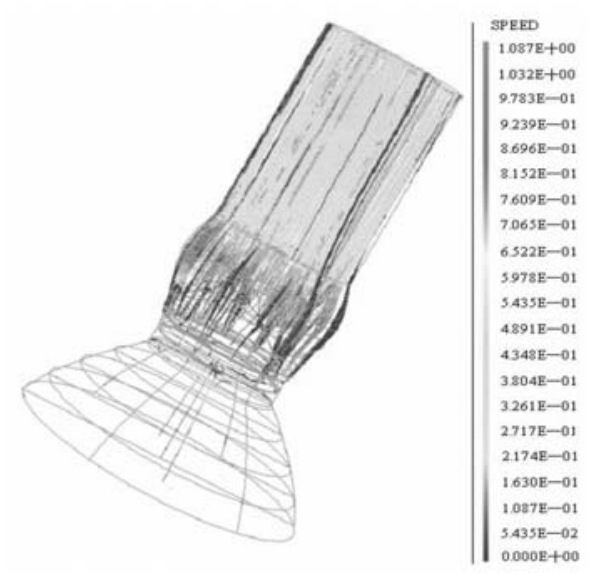

Fig 7. Streamline distribution of model-2 PU aortic valve

Table II Range of axial velocities of model-2 PU aortic valve at different sections

\begin{tabular}{|c|c|c|}
\hline Distance from inlet $(\mathrm{mm})$ & Maximum $w$ value $(\mathrm{m} / \mathrm{s})$ & Minimum $w$ value $(\mathrm{m} / \mathrm{s})$ \\
\hline 27.0 & 0.2700897 & $2.3920472 \mathrm{E}-07$ \\
\hline 31.6 & 0.1905822 & $0.0000000 \mathrm{E}+00$ \\
\hline 32.0 & 0.2053338 & $1.9708291 \mathrm{E}-07$ \\
\hline 33.0 & 0.2252978 & $3.9868783 \mathrm{E}-07$ \\
\hline 35.0 & 0.3750673 & $0.0000000 \mathrm{E}+00$ \\
\hline 39.0 & 0.2194727 & $5.1996067 \mathrm{E}-06$ \\
\hline 40.0 & 0.2217083 & $3.7694744 \mathrm{E}-07$ \\
\hline 47.7 & 0.3471988 & $4.9365627 \mathrm{E}-07$ \\
\hline
\end{tabular}

\section{CONCLUSION}

Table III compares the maximum velocity of model-1 and model-2 PU aortic valve at fully open condition with different inlet flow rate. At peak flow rate of $380 \mathrm{ml} / \mathrm{s}$, the improved model can effectively reduce the violent velocity change upstream the sewing ring. The maximum velocity of model-1 is $1.144 \mathrm{~m} / \mathrm{s}$ and it is reduced to $0.7609 \mathrm{~m} / \mathrm{s}$ for model- 2 with the reduction rate of $33 \%$.

Table III. Comparison of maximum velocity at different flow rates

\begin{tabular}{|c|c|c|c|}
\hline & Case $1(380 \mathrm{ml} / \mathrm{s})$ & Case $2(290 \mathrm{ml} / \mathrm{s})$ & Case $3(200 \mathrm{ml} / \mathrm{s})$ \\
\hline model-1 & $1.144 \mathrm{~m} / \mathrm{s}$ & $0.8734 \mathrm{~m} / \mathrm{s}$ & $0.6022 \mathrm{~m} / \mathrm{s}$ \\
\hline model-2 & $0.7609 \mathrm{~m} / \mathrm{s}$ & $0.6054 \mathrm{~m} / \mathrm{s}$ & $0.4809 \mathrm{~m} / \mathrm{s}$ \\
\hline velocity change rate & $-33 \%$ & $-30 \%$ & $-20 \%$ \\
\hline
\end{tabular}

At flow rate of $290 \mathrm{ml} / \mathrm{s}$, blood flow for the improved model is comparatively smooth-going and the largest velocity of flow is nearly reduced by $30 \%$. In the case of volume rate of $200 \mathrm{ml} / \mathrm{s}$ the largest velocity of flow is nearly reduced by $20 \%$.

After modifying the support height and fully opening effective area, the improved model can highly reduce the possibility of vortex induced compared with that of original model. The maximum reversed axial velocity changes from the original model of $-0.07 \mathrm{~m} / \mathrm{s}$ to almost zero of the improved model. This is predicted to decrease the possibility of thrombus formation or blood coagulation. Fig. 8 compares the vortex flow field of the original model with that smooth flow at the regime of the improved model.

With the flow field analysis of model- 1 and model-2 separately, we can roughly sum up the following several conclusions.

(1) At peak systole, the opening area of the original model is limited and will produce more violent velocity at upstream of the sewing ring entrance, especially when the flow rate of blood reaches peak value. Its largest speed is nearly up to $1.144 \mathrm{~m} / \mathrm{s}$. There exist large vortex flows in about $6 \sim 7 \mathrm{~mm}$ downstream the leaflet support top. The maximum reversed axial velocity reaches $-0.07 \mathrm{~m} / \mathrm{s}$ at the location.

(2) After modifying the PU aortic valve geometry, velocity change in the sewing ring area slows down obviously and flow field distributes smoothly and with stability. The maximum velocity at peak systole reduces to about $0.7609 \mathrm{~m} / \mathrm{s}$ and the corresponding reduction is nearly $33 \%$. There is no vortex flow and recirculation zone for the overall flow field. This highlights that the improved PU aortic valve model could effectively reduce vortex phenomenon and prevent thrombus formation and blood coagulation. 


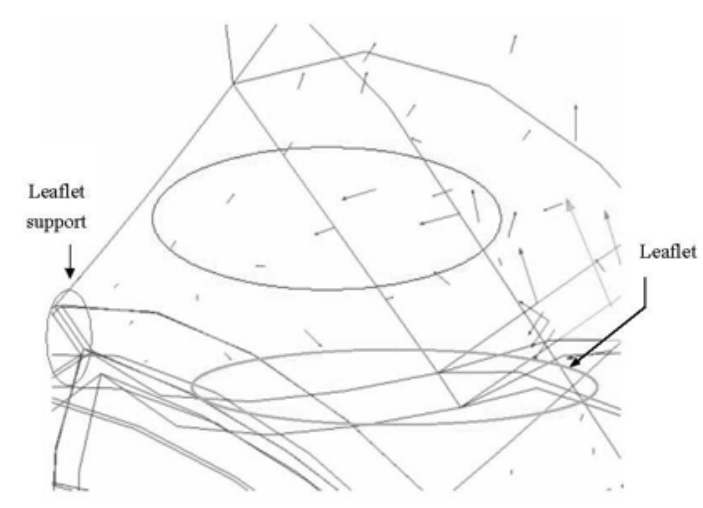

(a) Local flow region of model-1 PU aortic valve with vortex formation

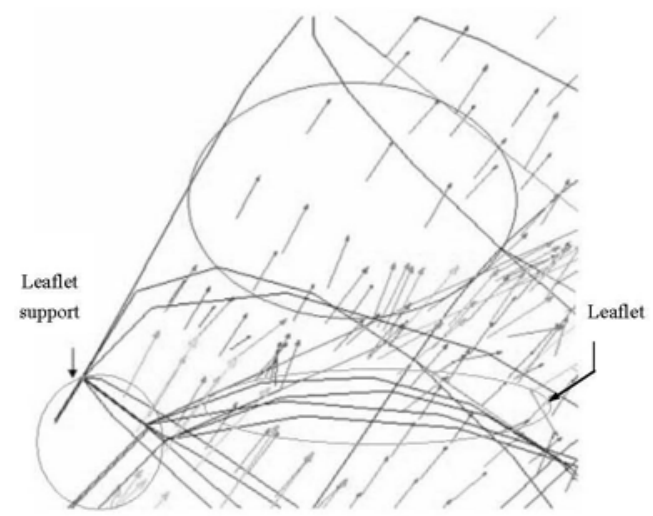

(b) Local flow region of model-2 PU aortic valve with no vortex

Fig 8. Comparison of the vortex flow field of the original model with that smooth flow at the improved model

\section{ACKNOWLEDGEMENT}

We do appreciate National Science Council R.O.C. with great supports for this work (grand number NSC 94-2614-E-218-001).

\section{REFERENCE}

1. Bloor, M. I. G.. Wilson, M. J. 1989. Generating blend surfaces using partial differential equations. Computer-Aided Design 21(3), 165-171.

2. Hsu, C. H. 1005. A visualization design environment for quick designs of prosthetic mechanical heart valves. Leeds University PhD Dissertation.
3. Zhang, M. J. Pomfret, M. J. Wong, C. M. 1996. Performance prediction of a backswept centrifugal impeller at off-design point conditions. International Journal for Numerical Methods in Fluids 23, 883895.

4. Corden, J. David, T. Fisher, J. 1996. The influence of open leaflet geometry on the hemodynamic flow characteristics of polyurethane trileaflet artificial heart valves. Proc. Inst. Mech. Engrs. (Part H), Engineering in Medicine 210, 273-287.

5. Takahama, T. Kanai, F. Onishi, K. 2000. Anticoagulation during use of a left ventricular assist device. ASAIO J 46, M354-M357.

6. Sbardella, S. Imregun, M. 2001. Linearized unsteady viscous turbo-machinery flows using hybrid grids. ASME J Turbo-machinery 123, 568-582. 